University of Wollongong

Research Online

Faculty of Engineering and Information

Faculty of Engineering and Information

Sciences - Papers: Part A

Sciences

2017

\title{
Reproducible bubble-induced acoustic microstreaming for bead disaggregation and immunoassay in microfluidics
}

\author{
Huaying Chen \\ CSIRO Manufacturing \\ Yuan Gao \\ CSIRO Manufacturing, yg984@uow.edu.au \\ Karolina Petkovic \\ CSIRO Manufacturing \\ Sheng Yan \\ University of Wollongong, sy034@uowmail.edu.au \\ Michael Best \\ CSIRO Manufacturing
}

See next page for additional authors

Follow this and additional works at: https://ro.uow.edu.au/eispapers

Part of the Engineering Commons, and the Science and Technology Studies Commons

Research Online is the open access institutional repository for the University of Wollongong. For further information contact the UOW Library: research-pubs@uow.edu.au 


\title{
Reproducible bubble-induced acoustic microstreaming for bead disaggregation and immunoassay in microfluidics
}

\author{
Abstract \\ The bead-based immunoassay requires not only efficient mixing but also good control of bead-surface- \\ area-to-sample-volume ratio to realise accurate and reproducible detection of low concentration samples. \\ This paper reports the development of a microfluidic platform with the reproducible and efficient bubble- \\ induced micromixing for bead disaggregation and immunoassay of prostate-specific antigen (PSA). The \\ platform consists of a microfluidic chip with a microchamber and rectangular traps for capturing air \\ bubbles and a home-made controller to generate sound wave using an external piezo transducer. \\ Methods for reproducible bubble formation and bubble size control during mixing are explored. The \\ influence of driving voltage, PDMS thickness and the substrate material on the mixing efficiency is \\ characterised by mixing a fluorescence dye and a buffer solution. The optimised acoustic microstreaming \\ is able to break clusters with hundreds of beads and homogenise individual beads over the \\ microchamber. Immunoassay with efficient micromixing has been applied to PSA immunoassay with \\ greatly reduced detection time. This study provides a practical guide for the design and development of \\ the bubble-induced acoustic micromixers for bead disaggregation and on-chip immunoassays.

\section{Disciplines} \\ Engineering | Science and Technology Studies

\section{Publication Details} \\ Chen, H., Gao, Y., Petkovic, K., Yan, S., Best, M., Du, Y. \& Zhu, Y. (2017). Reproducible bubble-induced \\ acoustic microstreaming for bead disaggregation and immunoassay in microfluidics. Microfluidics and \\ Nanofluidics, 21 (3), 30-1-30-14.

\section{Authors} \\ Huaying Chen, Yuan Gao, Karolina Petkovic, Sheng Yan, Michael Best, Yanping Du, and Yonggang Zhu
}


Reproducible bubble-induced acoustic microstreaming for bead disaggregation and immunoassay in microfluidics

Huaying Chen ${ }^{12}$, Yuan $\mathrm{Gao}^{2}$, Karolina Petkovic ${ }^{2}$, Sheng Yan $^{3}$, Michael Best ${ }^{2}$, Yanping $\mathrm{Du}^{2}$ and Yonggang $\mathrm{Zhu}^{* 124}$

${ }^{1}$ School of Mechanical Engineering and Automation, Harbin Institute of Technology

(Shenzhen), Shenzhen 518055, P.R. China

${ }^{2}$ CSIRO Manufacturing, Private Bag 10, Clayton, VIC 3168, Australia

${ }^{3}$ School of Mechanical, Materials and Mechatronic Engineering, University of Wollongong, Wollongong, NSW 2522, Australia

${ }^{4}$ School of Science, RMIT University Melbourne, VIC 3001, Australia

* Corresponding Author: zhuyonggang@hitsz.edu.cn 


\section{Abstract}

The bead-based immunoassay requires not only efficient mixing but also good control of bead-surface-area-to-sample-volume ratio to realize accurate and reproducible detection of low concentration samples. This paper reports the development of a microfluidic platform with the reproducible and efficient bubble-induced micromixing for bead disaggregation and immunoassay of prostate specific antigen (PSA). The platform consists of a microfluidic chip with a microchamber and rectangular traps for capturing air bubbles and a home-made controller to generate sound wave using an external piezo transducer. Methods for reproducible bubble formation and bubble size control during mixing are explored. The influence of driving voltage, PDMS thickness and the substrate material on the mixing efficiency is characterised by mixing a fluorescence dye and a buffer solution. The optimised acoustic microstreaming is able to break clusters with hundreds of beads and homogenize individual beads over the microchamber. Immunoassay with efficient micromixing has been applied to PSA immunoassay with greatly reduced detection time. This study provides a practical guide for the design and development of the bubble-induced acoustic micromixers for bead disaggregation and on-chip immunoassays.

\section{Key words}

Bubble, acoustic, mixing, piezo, microstreaming, immunoassay, PSA, lifetime, disaggregation 


\section{Introduction}

Magnetic bead-based immunoassays are the current gold standard for many biochemical tests in clinics and the discovery of new biomarkers in pharmaceutical and biological applications. However, conventional immunoassays are of limitations, such as consumption of large volume of sample and reagents, high cost, long analysis time and need for experienced personnel to perform the assays. This has prompted the development of microfluidics-based immunoassays(Veetil et al. 2010), since microfluidic devices have the advantages of low reagent consumption, faster reaction, lower cost and a much higher degree of automation. Additionally, these devices offer the possibility of integrating physical phenomena, such as magnetics, acoustics, and electrokinetics, with hydrodynamics for sample processing(Veetil et al. 2010). For example, magnetic forces have been extensively employed for trapping, separation, actuation (Pamme 2012; van Reenen et al. 2014) and micromixing (Riahi and Alizadeh 2012; Rida and Gijs 2004; Wang et al. 2008; Zolgharni et al. 2007) in bead-based immunoassay.

To increase the assay sensitivity and reproducibility of the magnetic bead-based immunoassay in microfluidics, aggregation of beads should be avoided and bead-surfacearea-to-sample-volume ratio is required to be well controlled. Magnetic beads tend to form chains or clusters following the introduction of the magnetic field. The number of beads in a chain or a cluster is uncontrollable, so the accessibility of bead surface can be dramatically reduced. Therefore, the reproducibility and sensitivity of the assay can be significantly affected by the uncontrollable bead-surface-area-to-sample-volume ratio, especially when the concentration of target analyte is low. To overcome this problem and acquire individual beads, Gao et.al (Silvestre et al. 2013) proposed a magnetic force based disaggregation 
technique using time-dependent magnetic fields generated by eight external electromagnets. This technique can successfully disperse several tens of particles within about one minute, but the complexity limits its application and miniaturization.

When low concentration analyte is of interest, efficient and reproducible mixing of beads and reagents in the microfluidics is critical for reducing the detection time and increasing the sensitivity. A number of techniques have been developed to enhance sample mixing in microfluidics in the past two decades. These techniques can be classified into passive mixing, such as staggered herringbone mixers (Stroock et al. 2002), and active mixing, such as electrokinetic (Oddy et al. 2001), magnetic (Bau et al. 2001; Kitenbergs et al. 2015) and acoustic (Gao et al. 2015; Petkovic-Duran et al. 2009) mixers. These mixers have been reviewed in previous literature (Capretto et al. 2011; Kevin and Fan 2015; Lee et al. 2011). Among the active mixing techniques, acoustic micromixing has attracted considerable interests (Ahmed et al. 2009a; Liu et al. 2002), since a) its efficiency is high and reagents can be fully mixed in seconds or even milliseconds (Ahmed et al. 2009a), and b) the mixing is non-invasive (no micro magnetic beads are required in comparison with magnetic mixing).

The acoustic micromixing can be implemented either with or without bubbles in the mixing channels or chambers. For non-bubble acoustic mixing, a high driving frequency in the range of megahertz is usually required. For example, micro or miniaturized transducers were integrated inside or on both sides of a microchannel to mix two parallel laminar-flow streams (Guo et al. 2008; Yaralioglu et al. 2004). In addition, microelectrodes were used to generate surface acoustic wave to achieve mixing in a microchannel (Jo and Guldiken 2013) or a microwell (Shilton et al. 2011). Bubbles were firstly employed in microfluidics to enhance acoustic mixing by Liu et al. (Liu et al. 2002). In the last decade, it has been extensively applied in various applications, such as cancer biomarker detection (Lin et al. 2014), PCR (Liu et al. 2004), as well as particle and cell separation (Patel et al. 2014; Patel et al. 2012). 
Huang et al. reported the ultra-fast mixing of two liquid streams flowing side-by-side in a microchannel using staggered and pre-trapped air bubbles along the side walls of the channel and a piezo transducer glued in the vicinity of the channel (Ahmed et al. 2009a). In another report (Ahmed et al. 2009b), in-line ultrafast mixing was achieved by a single bubble retained in a horse-shoe shaped trap in the midplane of a microchannel. Additionally, an array of bubbles was employed to generate microstreaming, which significantly reduces the mixing time from hours to seconds for a DNA microarray hybridization (Liu et al. 2003).

In addition to the inherent advantages of the acoustic mixing, the bubble-induced acoustic mixing device is simple in fabrication due to the application of the piezo transducer. Additionally, the presence of air/liquid interface not only allows fast mixing at low driving frequencies but also provides the flexibility of predefining the location for mixing at the microscale. Low frequency is favorable for the miniaturization of the system, since it can be generated using a piezo transducer driven by a simple electronic board instead of a function generator which is indispensable for the non-bubble acoustic mixing. Furthermore, the air bubbles can be trapped either in the inlet channel of two fluid streams (Ahmed et al. 2009b) or around the edge of mixing chambers (Liu et al. 2003) to confine the locations of the mixing. This is particularly important for lab-on-a-chip platforms integrating multiple components and functions (Liu et al. 2003).

Several factors may affect bubble-induced mixing activated by a piezo transducer. Mixing reproducibility and efficiency are dependent on bubble size, which affects the resonance frequency of bubbles. Fan et al. (Huang et al. 2015) reported that bubbles captured by multiple traps around a chamber can differ by $15 \%$ in size. In order to achieve reproducible mixing, trapping bubbles with consistent size and controlling their size during the whole mixing period are critical. In addition to bubble size, other factors such as the driving frequency, the location of the piezo, and the chip materials are also important to maximize 
mixing efficiency. However, there have been no detailed studies on these factors.

The main objective of this study is to develop a microfluidic platform which utilizes bubbleinduced microstreaming for bead disaggregation and on-chip immunoassays. The bubble size, driving voltage and frequency of the piezo, thickness of the PDMS chip, and the substrate material are evaluated in order to determine optimal conditions to achieve reproducible microstreaming. The microstreaming is then employed to break and homogenize clusters with hundreds of magnetic microbeads over the microchamber. The microstreaming is demonstrated to significantly reduce the detection time of the bead-based immunoassay for prostate specific antigen. This study provides a practical guide for the design and application of the reproducible bubble-induced acoustic microstreaming in immunoassay within microfluidics.

\section{Materials and methods}

\subsection{Microfluidic chip design}

Microfluidic chips (Figure 1A) with a microchamber and bubble traps are developed to mix nanolitres of fluids using bubble-induced acoustic microstreaming. The microstreaming is induced by the sound field generated by a piezo transducer (referred to as piezo hereafter) attached to the microfluidic chip. The microfluidic chip consists of a PMDS layer with microchannels, a mixing microchamber and bubble traps (Figure 1B and C). The mixing microchamber is $1 \mathrm{~mm}$ in diameter. It is linked with either two rectangular bubble traps with both the length and the width of $400 \mu \mathrm{m}$ (referred to as the two-bubble device, Figure 1B) or three traps with the length of $400 \mu \mathrm{m}$ and the width of 100, 200 and $400 \mu \mathrm{m}$ (referred to as the three-bubble device, Figure 1C). The traps are evenly placed around the microchamber edge. A glass or PDMS capping layer is used to seal the top PDMS layer. The depth of the channels, microchamber and bubble traps is $100 \mu \mathrm{m}$. The volume of the microchamber is 
approximately $78.5 \mathrm{~nL}$. The bubble traps retain air bubbles with defined volumes, when phosphate buffer solution (PBS) is injected into the empty microchamber. A piezo is placed on top of the PDMS layer (above the microchamber). A home-made controller drives the piezo at the desired frequency to induce acoustic microstreaming near the bubble interfaces. The resonance frequencies of rectangular air bubbles in water are given by the equation reported by Chindam et al. (Chindam et al. 2013), and are found to be $12.6,8.9$ and $7.9 \mathrm{kHz}$ for bubbles with the width of 100, 200 and $400 \mu \mathrm{m}$, respectively.

\subsection{Microchip fabrication}

The microfluidic chips were manufactured at the Microfluidics Laboratory at CSIRO and the Melbourne Centre for Nanofabrication. The moulds (Figure 1D) were fabricated using standard photolithography of SU-8 as described elsewhere (Chen et al. 2011; Chen et al. 2012; Hai et al. 2009). A 'clamp casting' technique was applied to produce PDMS layers with the thickness of $0.5 \mathrm{~mm}, 1 \mathrm{~mm}$ and $2 \mathrm{~mm}$ between a glass slide and a silicon wafer (Figure 1DG). Firstly, Sylgard ${ }^{\circledR} 184$ base and the curing agent (PDMS, Dow Corning) were mixed with the weight ratio of 10:1 and cast against the SU-8 mould (Figure 1E). Following degassing, a glass slide (75 × $50 \times 1 \mathrm{~mm}$, see Figure $1 \mathrm{~F}$ ) attached with four spacers (measuring $8 \times 8 \mathrm{~mm}$, the thickness of $0.5,1$ or $2 \mathrm{~mm}$ ) was pressed into the uncured PDMS till the spacer formed conformal contact with the wafer. Then the top glass slide and the bottom silicon wafer were clamped together (Figure $1 \mathrm{G}$ ). After baking at $60^{\circ} \mathrm{C}$ for $2 \mathrm{~h}$, the PDMS replica was cut off from the mould, punched with inlet and outlet ports, and bonded to either a glass slide or a 1mm thick blank PDMS layer following 2-min air plasma etching. Finally, the device was connected to microbore PTFE tubing (Cole-Parmer ${ }^{\circledR}$, inner diameter: $300 \mu \mathrm{m}$ ) and baked overnight at $60^{\circ} \mathrm{C}$ before further use. 


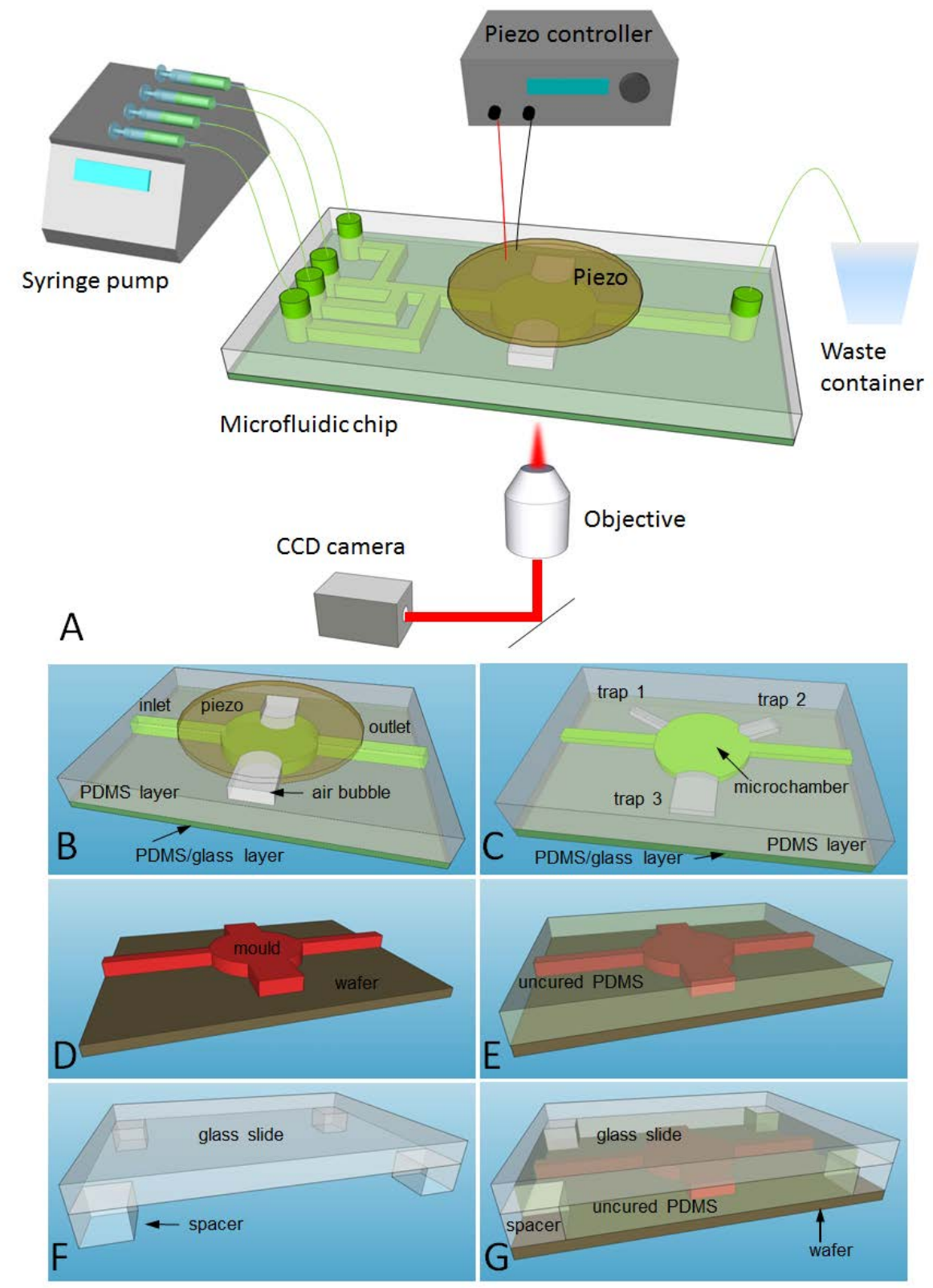

Figure 1 A schematic drawing of the experiment setup and the fabrication process of the microchip. A) The schematic drawing of the experiment system. B) The two-bubble device with a piezo placed above the microchamber. Both length and width of the traps are $400 \mu \mathrm{m} . \mathrm{C})$ The three-bubble device. The length of all traps was $400 \mu \mathrm{m}$. The width of trap 1, 2 and 3 was 100, 200 and $400 \mu \mathrm{m}$, respectively. D) The SU-8 mould on the silicon wafer. E) Uncured PDMS was casted on the mould. F) A glass slide was attached with four spacers (either 0.5, 1 or $2 \mathrm{~mm}$ thick). G) The glass slide with spacers was pressurised into uncured PDMS.

\subsection{Piezo controller}

An electronic controller based on an Arduino ELEVEN R3 v2.0 (Freetronics) board was developed to activate the piezo with desired frequency and voltage. The digital output of the pulse width modulation on the board was used to generate the square-shaped wave with the 
duty cycle of $70 \%$ and the driving frequency ranging from 0 to $30 \mathrm{kHz}$. An external DC power supply provided the voltage up to $30 \mathrm{~V}$ across the piezo. The piezo (element 14) with the resonance frequency of $9 \mathrm{kHz}$ was connected to the controller and attached to the PDMS layer using the ultrasound gel (see Figure 1A).

\subsection{Bubble formation study}

Both two-bubble and three-bubble devices (Figure 1B and C) were employed. Phosphate buffer solution (PBS) was injected into the empty microchamber at the flow rate of $0.01,0.1$, $1,5,10,15$ or $25 \mu \mathrm{L} / \mathrm{sec}$. The air bubbles formed at various flow rates were imaged using a PCO Sensicam digital camera and measured using MATLAB image processing code.

\subsection{Bubble size study}

The variation of bubble size in one hour after formation was studied in the three-bubble device either with or without acoustic mixing. After bubble trapping at the flow rate of 1 $\mu \mathrm{L} / \mathrm{sec}$, the fluorescent polystyrene beads (Fluoro-Max ${ }^{\mathrm{TM}}, 1 \mu \mathrm{m}$ in diameter, $1 \%$ solid) were diluted 200 fold in PBS solution and manually injected into the microchamber for visualising the mixing flow. The outlet tubing was either open to the atmosphere or connected to a water container raised for 0-75 $\mathrm{cm}$ to generate hydraulic pressure of 0-735 $\mathrm{Pa}$. The piezo was then activated at $12 \mathrm{~V}$ and $8 \mathrm{kHz}$ to induce streaming flow in the microchamber. The bubbles and the acoustic streaming patterns were imaged using a $4 \times$ objective lens every 1 min for 60 min.

\subsection{Parametric study of mixing}

Following the formation of bubbles at the flow rate of $1 \mu \mathrm{L} / \mathrm{sec}$, the diluted beads (Fluoro$\mathrm{Max}^{\mathrm{TM}}$ ) were injected into the microchamber using a micropipette. When the liquid flow was stopped and beads were free of flow-induced motion, the piezo was activated at frequencies from 2 to $14 \mathrm{kHz}$ with the increment of $1 \mathrm{kHz}$. The acoustic-induced flow of microbeads at each frequency was imaged with the exposure time of 3 seconds. 
Mixing in the two-bubble device was characterised when the driving voltage, the thickness of the top PDMS layer and the substrate material (PDMS vs glass) were varied. After the bubble formation at the injection flow rate of $1 \mu \mathrm{L} / \mathrm{sec}$, the fluorescent dye (Alexa Fluor ${ }^{\circledR} 488$ ) and the PBS with $0.1 \%$ Triton X-100 (PBST) were simultaneously injected into the microchamber from the two inlet ports at the flow rate of $0.02 \mu \mathrm{L} / \mathrm{sec}$. After the flow became stable, the injection was stopped and the piezo was activated immediately at the defined frequency and voltage. The mixing process was imaged every 2 seconds with the exposure time of 800 milliseconds.

\subsection{Disaggregation of beads}

Disaggregation of magnetic microbeads in the microchamber was studied to demonstrate the ability of acoustic microstreaming to break bead clumps into individual beads. A two-bubble device with 1-mm thick PDMS top layer on a glass bottom was employed. The SPHEROTM carboxyl magnetic microbeads (Spherotech, Inc., 5-5.9 $\mu \mathrm{m}$ ) were diluted in PBST solution with the volume ratio of 1:20. Following the formation of air bubbles at the flow rate of 1 $\mu \mathrm{L} / \mathrm{sec}$, the bead solution was injected into the microchamber using a micropipette. Then magnets were placed on the side of one trap. After most beads migrated to the vicinity of the bubble/air interface due to the external magnetic field, the magnets were removed. Then acoustic induced flow was activated at the driving voltage of $30 \mathrm{~V}$ and the frequency of $5 \mathrm{kHz}$. The disaggregation process was imaged every 1 second with a $4 \times$ objective lens.

\subsection{On-chip immunoassay}

The magnetic beads were conjugated to a prostate specific antigen (PSA) capture antibody using EDC/NHS chemistry and the PSA detection antibody was conjugated to the lightningLink R-PE conjugation kit (R-PE label) (Wang et al. 2014). 
The micromixing at optimised conditions was applied to demonstrate its ability to enhance biomarker detection using immunoassay. The two-bubble device with 1-mm thick PDMS top layer on a glass bottom was applied in this study. Following the formation of air bubbles at the flow rate of $1 \mu \mathrm{L} / \mathrm{sec}, 1 \mu \mathrm{L}$ magnetic beads (conjugated to the PSA capturing antibody) were injected into the microchamber using a micropipette and retained in situ by magnets placed $1 \mathrm{~mm}$ above the top PDMS layer. PSA solution (either $100 \mathrm{ng} / \mathrm{mL}$ or $5 \mathrm{ng} / \mathrm{mL}$ ) was injected into the microchamber at $0.05 \mu \mathrm{L} / \mathrm{sec}$ for 30 seconds. Afterwards, the solution in the microchamber was either kept static (referred to as 'Static incubation') or mixed (referred to as 'Mixing') at $5 \mathrm{kHz}$ and $12 \mathrm{~V}$ for 5,15 or 30 minutes after the magnets had been removed. Then beads were retained in the microchamber by magnetic field and washed using PBST for 2 minutes at the flow rate of $0.05 \mu \mathrm{L} / \mathrm{sec}$. The R-PE label was then injected into the microchamber at the same flow rate for 30 seconds, before being incubated statically or mixed using the microstreaming for 5 minutes without external magnetic field. Finally, the beads were retained by the magnets, washed as above and imaged using a $20 \times$ objective lens.

\subsection{Image processing and data analysis}

The images were analysed using the MATLAB Image Processing Toolbox and the Image $\mathrm{J}$ (Schneider et al. 2012). The standard deviation of the fluorescence intensity (SDFI) of pixels in the microchamber from the dye mixing study was used to characterise the mixing efficiency. The data were analysed using the one-way ANOVA with the significance level of 0.05. The results presented in this study are the mean ( \pm standard deviation) of three independent replicates. 


\section{Results and discussion}

\subsection{Bubble formation study}

Figure 2A shows the formation of bubbles in three traps at the flow rate of $0.01 \mu \mathrm{L} / \mathrm{sec}$ (see Supplementary video 1 for details). When the PBS was pumped into the empty microchamber, the air/liquid interface was pushed downstream. When the liquid reached the second corner of a trap, a pocket of air (air bubble) was captured. Figure 2B shows the micrograph of bubbles formed immediately after the injection of PBS at the flow rate of $25 \mu \mathrm{L} / \mathrm{sec}$. It is obvious that the bubbles are much bigger than the relevant traps and cover large area of the microchamber, which is unfavourable for the mixing and following immunoassay (discussed below).

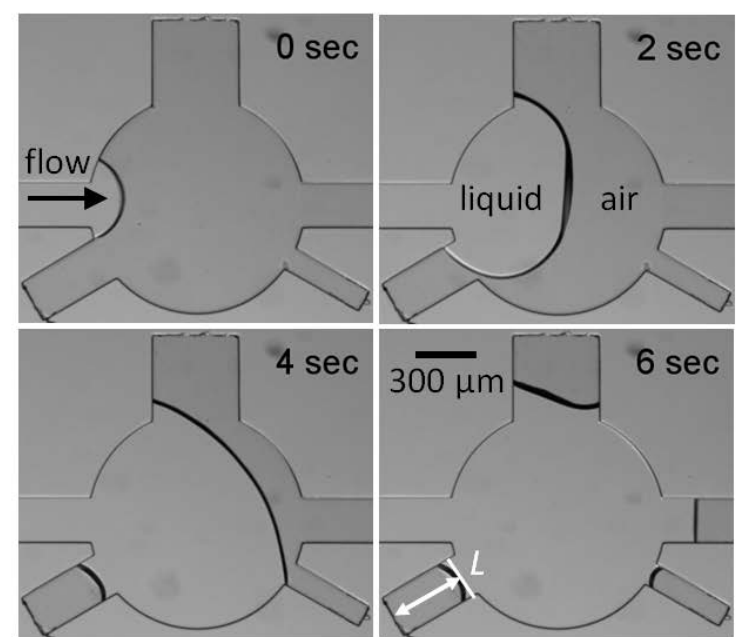

A

Weber number $=3.5 \times 10^{-7}$

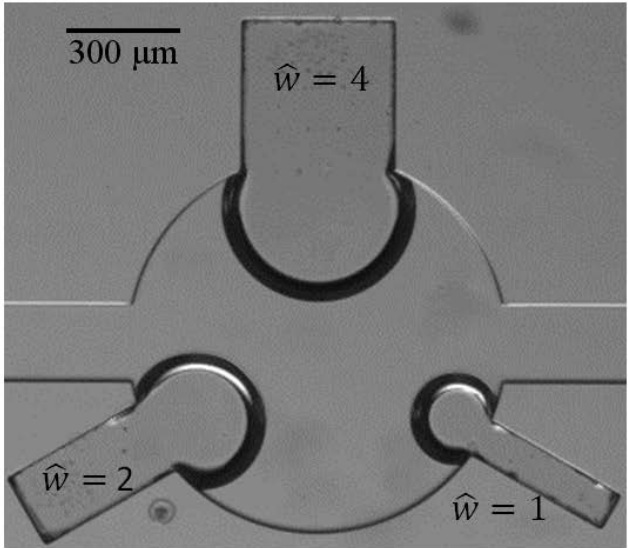

B Weber number $=2.17$

Figure 2 Micrographs of air bubbles. A) Formation of air bubbles when PBS was injected into the empty microchamber at the flow rate of $0.01 \mu \mathrm{L} / \mathrm{sec}$. B) The bubbles formed immediately at the flow rate of $25 \mu \mathrm{L} / \mathrm{sec}$.

Reproducible bubble length is critical for achieving stable bubble-induced microstreaming. Bubble length refers to the maximal distance from the end of the bubble trap to the air/liquid interface as shown by the white double-headed arrow in Figure 2A. Bubble length is a function of the device geometry and fluid properties:

$$
L=f(w, a, b, d, \rho, \sigma, \bar{v})
$$


where $L$ is the bubble length, $w$ is the width of the trap, $a$ and $b$ are the width and height of the inlet channel, respectively, $d$ is the diameter of the microchamber, $\rho$ and $o$ are the density and the surface tension of the fluid at room temperature, and $\bar{v}$ is the mean velocity at the inlet channel. For the current study, PBS and air are the liquid and gas phase, respectively. Equation (1) can be non-dimensionalised to:

$$
\frac{L}{w}=f\left(\frac{a}{w}, \frac{b}{w}, \frac{d}{w}, \frac{\rho \bar{v}^{2} w}{\sigma}\right)
$$

Therefore, the relevant dimensionless parameters are $\hat{L}=\frac{L}{u}, \hat{a}=\frac{a}{u} \hat{b}=\frac{b}{w}, \hat{d}=\frac{d}{w}$ and $W e=\frac{\rho \bar{v}^{2} w}{\pi}$. Since the interface between the PBS and air dictates the bubble formation process, the Weber number (We), which is the ratio of the inertia force to the surface tension, is an important factor here. The density $(\rho)$ of PBS is approximately $1000 \mathrm{~kg} / \mathrm{m}^{3}$. It is assumed that the surface tension ( $a$ ) of PBS solution is the same as that $\left(7.197 \times 10^{-2} \mathrm{~N} / \mathrm{m}\right)$ of water in air at room temperature. The width (a) and height $(b)$ of the inlet channel are $200 \mu \mathrm{m}$ and $100 \mu \mathrm{m}$, respectively, while the width (w) of the trap is $100 \mu \mathrm{m}, 200 \mu \mathrm{m}$ and $400 \mu \mathrm{m}$, respectively. The diameter of the microchamer is $1 \mathrm{~mm}$. The mean velocity at the inlet channel is in the range of $5 \times 10^{-4}-1.25 \mathrm{~m} / \mathrm{sec}$. For the current experimental conditions, We varies from $3.47 \times 10^{-7}$ to 2.17 . In this large range of $W e$, the inertial force contributes differently to the bubble formation.

Figure 3A-C show the dimensionless length of the bubbles captured in the three traps at various Weber numbers. Although the width of the bubbles is always equal to the trap width, their length is greatly affected by the Weber number. The bubble length remains almost constant and is close to the trap length at very low Weber numbers (between $3.5 \times 10^{-7}$ and 0.35 ) ( $p=0.53,0.7,0.83$ for the 100,200 , and $400 \mu \mathrm{m}$ wide trap, respectively). This is not surprising, since at low Weber numbers, the surface tension instead of the flow velocity dictates the size of the bubble. On the other hand, at high Weber numbers (i.e. 0.78 and 2.17), 
the flow rate contributes to the formation of the bubbles. Due to the fast-moving liquid, more air is trapped, so the bubbles longer than the traps. The large bubbles in neighbouring traps may merge together within one hour after bubble formation. Therefore, bubbles larger than their traps should be avoided.
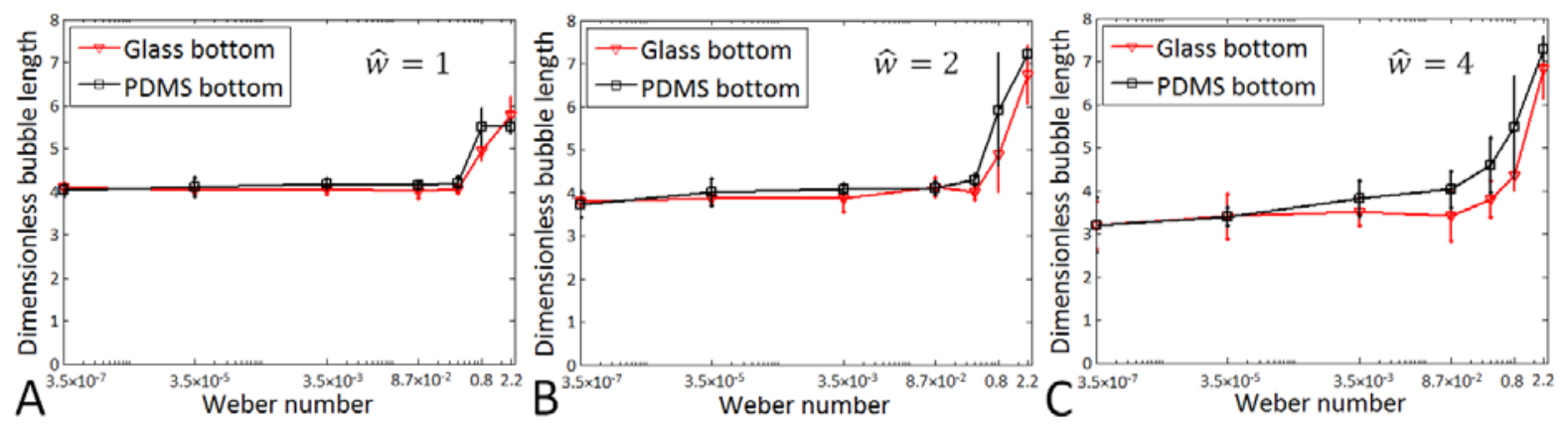

Figure 3 Effect of Weber number and trap width on the bubble size. The dimensionless length $(\widehat{L})$ of bubbles in

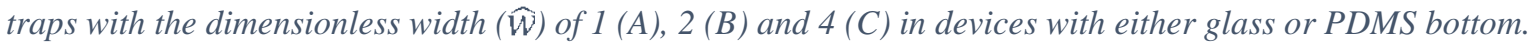

The surface property of the substrate material also affects the bubble formation due to their wettability difference. When the Weber number is larger than 0.35 , the size of bubbles on glass substrates is significantly bigger than that on PDMS substrates ( $p \approx 0.005-0.046$ ). However, this effect is not observed at lower Weber number $\left(3.5 \times 10^{-7}-0.087\right)(p>0.05)$. This may be related to the combination of Weber number and the contact angle of the substrate. Since PDMS is more hydrophobic than the glass. When the flow rate is high, the fluid cannot have enough time to wet or fill the whole microchamber. As a result, although the Weber number on both substrates is the same (see Figure 3), the bubble on PDMS is slightly larger than that on glass.

Since the acoustic streaming patterns are significantly affected by the shape and the position of the air/bubble interface, a reliable control for the formation of bubbles is crucial. When a bubble is much shorter than the trap, the acoustic streaming may not be strong or big enough to mix the samples in the microchamber (see details in Section 3.2). However, if a bubble is significantly longer than the trap, there are at least two drawbacks: a) the bubble width cannot 
be defined by the trap, so the resonance frequency may vary significantly. Thus the mixing efficiency at a predefined driving frequency may not be optimal; b) the bubbles occupy excessively large regions of the microchamber, which may greatly affect the assay by changing the sample volume. The optimal Weber numbers for trapping bubbles with reproducible sizes are suggested in the range of $3.5 \times 10^{-7}$ to 0.087 , regardless of the trap width and the substrate materials. For all the remaining studies, the Weber number of $3.5 \times$ $10^{-3}$ (the flow rate of $1 \mu \mathrm{L} / \mathrm{sec}$ ) was employed to generate air bubbles.

\subsection{Bubble size study}

This study aims to i) ascertain the influence of bubble size on acoustic streaming and ii) determine the optimal trap width and hydraulic pressure range to keep the bubble size as stable as possible for at least one hour (see Supplementary video 2). This time window is long enough for reproducibility and accuracy of the on-chip immunoassay. After formation, the bubbles were monitored for 60 minutes while various hydraulic pressures were applied to the outlet port. Figure 4A-D show the micrographs of both bubbles (the dark regions between the boundary of the trap and the green pathlines) and the acoustic streaming patterns (green pathlines) either 1 or 60 minutes following the bubble formation when the hydraulic pressure was set to either 0 (Figure $4 \mathrm{~A}$ and B) or $294 \mathrm{~Pa}$ (Figure $4 \mathrm{C}$ and D). The acoustic streaming was driven at $8 \mathrm{kHz}$ and $12 \mathrm{~V}$. At zero hydraulic pressure (Figure 4A and B), all bubbles were observed to expand in 60 minutes after formation. As a consequence, the microstreaming significantly decreased as indicated by the much smaller and less intense circulating flow patterns. At a hydraulic pressure of 294 Pa (Figure 4 C and D), the bubble size remained almost unchanged for the $200 \mu \mathrm{m}$ and $400 \mu \mathrm{m}$ traps during the 1-hour observation period. However, for the $100 \mu \mathrm{m}$ trap, the bubble shrunk into the trap as a result of the hydraulic pressure. The microstreaming gradually faded during this period. 

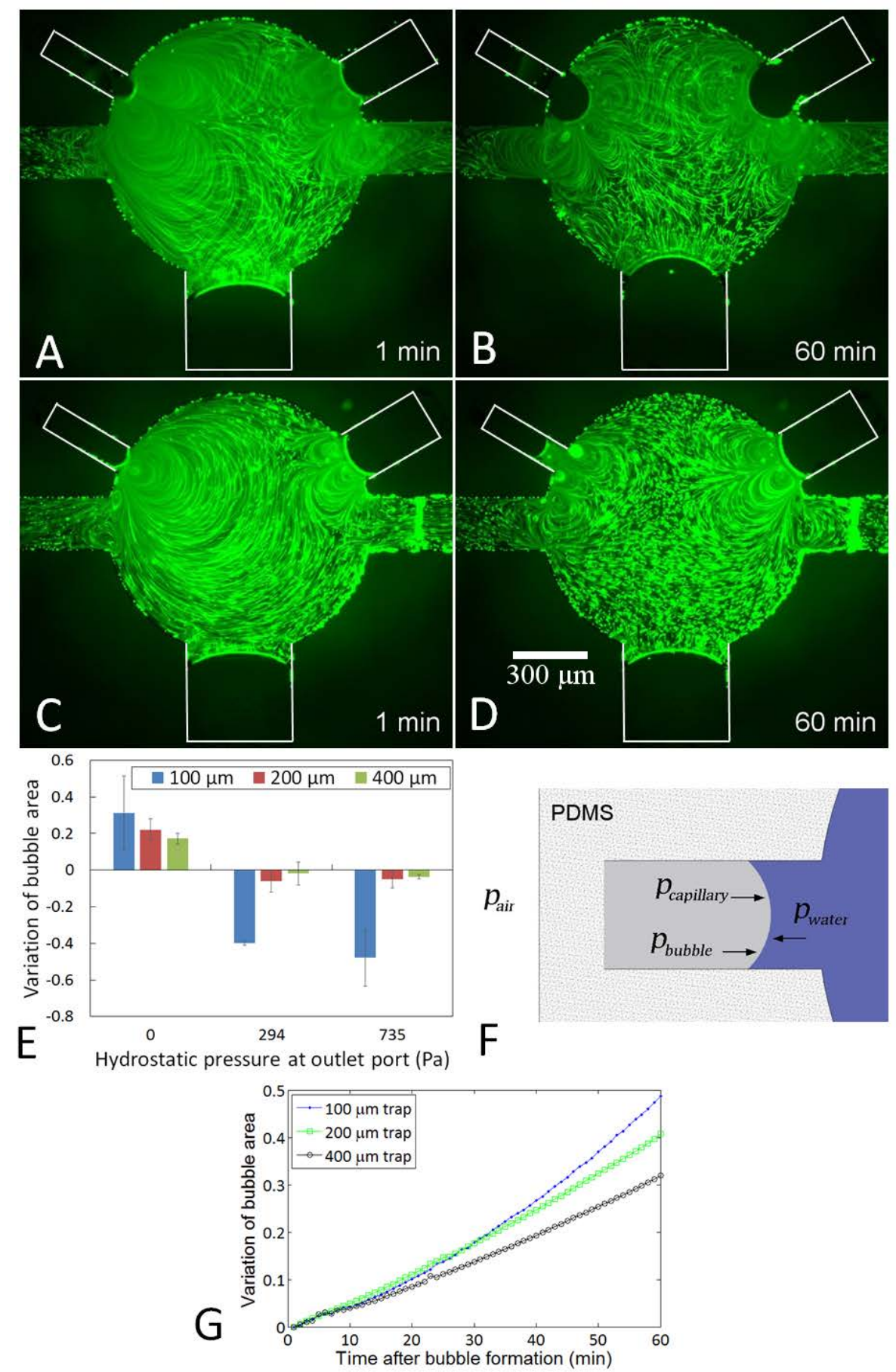

Figure 4 Micrographs of acoustic streaming patterns and bubble size variation. Acoustic streaming and air bubbles 1 and 60 minutes after bubble formation with the hydraulic pressure of 0 (A and B) and 294 (C and D) $P a$. The white lines highlight the boundary of bubble traps. The flow in all images was driven at the frequency of $8 \mathrm{kHz}$ and voltage of 12V. (E) Variation of bubble area in one hour after formation when the hydraulic pressure varied. (F) A schematic drawing showing the pressure balance of a trapped bubble. (G) Variation of bubble area as a function of time.

Figure 4E shows the variation of the bubble area in an hour after formation in the three traps

when the hydraulic pressure is 0, 294 and $735 \mathrm{~Pa}$, respectively. The variation is quantified by $\left(A_{t}-A_{0}\right) / A_{0}$, where $A_{t}$ is the bubble area at the time $t$, and $A_{0}$ is the bubble area immediately 
after bubble formation $(t=0)$. When the hydraulic pressure was zero, no matter if the mixing was present or not, the area of the 100, 200 and $400 \mu \mathrm{m}$ wide bubbles expanded by approximately 31\%, 22\% and 17\%, respectively. At the hydraulic pressure of $294 \mathrm{~Pa}$, the 100 $\mu \mathrm{m}$ bubble decreased by $40 \%$, while the $200 \mu \mathrm{m}$ and $400 \mu \mathrm{m}$ bubbles decreased by only $5.8 \%$ and $1.7 \%$, respectively. When the hydraulic pressure was further increased to $735 \mathrm{~Pa}$, the area of the $100 \mu \mathrm{m}$ wide bubble decreased by $48 \%$, while the area of the $200 \mu \mathrm{m}$ and 400 $\mu \mathrm{m}$ bubbles did not change significantly. From this study, it can be concluded that a) the 100 $\mu \mathrm{m}$ bubble is sensitive to the positive pressure and difficult to be controlled; and b) the 200 $\mu \mathrm{m}$ and $400 \mu \mathrm{m}$ wide bubbles are reliably controlled by hydraulic pressure in the range from 294 to $735 \mathrm{~Pa}$. Therefore, the two-bubble devices were selected for the on-chip immunoassay. The expansion and the shrinkage of a trapped air bubble are related to both pressures subjected by the air bubble and air mass transfer between bulk PDMS and the bubble (Volk et al. 2015). Kang et al. applied an exponential decay model to describe the air bubble elimination in a microfluidic device (Kang et al. 2008). As shown in Figure 4F, after PBS injection, the pressure inside and outside the bubble is in equilibrium. The pressure balance for the bubble is given below

$$
p_{\text {bubble }}=p_{\text {water }}+p_{\text {capillary }}
$$

where $p_{\text {hubhle }}$ is the air pressure in the bubble; $p_{\text {capillary }}$ is the capillary pressure and $p_{\text {water }}$ is the hydrostatic pressure of the PBS. Capillary pressure ( $\left.p_{\text {capillary }}\right)$ is given below (Delamarche et al. 1998):

$$
p_{\text {capillary }}=\sigma\left(\frac{2 \cos \theta_{1}}{w}+\frac{\cos \theta_{1}+\cos \theta_{2}}{h}\right)
$$

where $\sigma$ is the interfacial tension between PBST and air; $\theta_{1}$ is the contact angle for the left, right and top wall (PDMS substrate); $\theta_{2}$ is the contact angle for the floor (either PDMS or 
glass); and $w$ and $h$ are the width and height of the bubble. For devices with the PDMS bottom, $\theta_{1}=\theta_{2}$ :

When the air pressure $\left(p_{\text {buh hl }}\right)$ in a bubble is smaller than the atmosphere pressure $\left(p_{\text {oir }}\right)$ outside the PDMS layer, air permeates through PDMS layer into the bubble (Volk et al. 2015), which results in the increase of the bubble volume. Since a) the bottom of the device is either a glass slid or a PDMS slab on a glass slide and b) PDMS on side walls of the bubble is much thicker than the top PDMS layer, it is a reasonable assumption that the air permeation through the bottom and side walls of the bubble is negligible. Therefore, the bubble volume variation rate is given by

$$
\frac{d V}{d t}=J A
$$

where $V$ is the bubble volume; $t$ is time; $\mathrm{J}$ is the gas flux permeated from PDMS to the bubble; and $A$ is the projected area of the bubble. The flux was given by

$$
J=-\frac{P\left(p_{b}-p_{a}\right)}{\delta}
$$

where $P$ is the permeability coefficient, $p_{h}$ and $p_{a}$ are the pressure of the bubble and the atmosphere, $\delta$ is the thickness of the PDMS layer.

From Equations (5) and (6), the bubble area is given by the following exponential growth function of time $(t)$ :

$$
A(t)=a e^{b t}+c,
$$

where $a, b=-\frac{P\left(p_{b}-p_{a}\right)}{\delta h}$ and $c$ are constants; $t$ is time after bubble formation. Assuming the pressure difference $\left(p_{\text {hy hh? }}-p_{\text {oir }}\right.$ ) between the air bubble and outside atmosphere is constant, the model becomes the first order exponential growth or decay model. It is able to predict 
both the expansion (when $p_{\text {huhble }}<p_{\text {air }}$ ) and the shrinkage (when $p_{\text {humble }}>p_{\text {air }}$ ) of air bubbles in microfluidic devices.

Figure $4 \mathrm{G}$ shows the variation of the area of 100,200 , and $400 \mu \mathrm{m}$ bubbles as a function of time when the hydraulic pressure is zero and the acoustic mixing is absent. The area of all bubbles increased with time. The narrow bubbles have greater area increase rate in comparison to the wide bubbles. The area is well described by the exponential growth model $\left(\mathrm{R}^{2}>0.9996\right)$ in Equation (7). The constants $a, b$, and $c$ for the model of the $100 \mu \mathrm{m}$ wide bubble are $1.309 \times 10^{4}( \pm 860), 0.01709\left( \pm 7 \times 10^{-4}\right)$ and $3.35 \times 10^{4}( \pm 930)$, respectively. The constants $a, b$, and $c$ for the model of $200 \mu \mathrm{m}$ wide bubble are $5 \times 10^{4}( \pm 3290), 8.862 \times 10^{-3}$ $\left( \pm 4.52 \times 10^{-4}\right)$ and $3.52 \times 10^{4}( \pm 3370)$, respectively. The constants $a, b$, and $c$ for the model of $400 \mu \mathrm{m}$ wide bubble are $7.908 \times 10^{4}( \pm 8430), 8.59 \times 10^{-3}\left( \pm 7.15 \times 10^{-4}\right)$ and $8.628 \times 10^{4}$ ( \pm 8620$)$, respectively.

\subsection{Parametric study of mixing}

In addition to the reproducibility of the bubble size, the acoustic mixing efficiency is another critical requirement for the application of bubble-induced microstreaming in immunoassay. The microstreaming was visualized by imaging the pathlines of fluorescent microbeads with the exposure time of 3 seconds. The acoustic streaming pattern (as indicated by the pathlines in Figure 4A-D) was used to qualitatively identify the optimal driving frequencies to acquire the fastest flow. For a given exposure time, the faster the acoustic-induced flow is, the bigger the acoustic streaming pattern is. It was confirmed that, for the given device and driving voltage, when the driving frequency was approaching 5-6 kHz, the acoustic streaming pattern became bigger and reached maximum at $5-6 \mathrm{kHz}$. Therefore the velocity of the acousticinduced flow was maximal when the driving frequency was 5-6 kHz. This frequency was smaller than the theoretical resonance frequency $(7.9 \mathrm{kHz})$ of the $400 \mu \mathrm{m}$ wide rectangular 
bubble calculated using the equation in literature (Chindam et al. 2013). This might be related to the shape of the air/liquid interface. It was circular in this study but straight in the literature (Chindam et al. 2013). For the dye mixing characterisation and on-chip PSA immunoassay, the driving frequency of $5 \mathrm{kHz}$ was used.

The mixing of fluorescent dye and PBST in a two-bubble device was characterized to study the effect of the driving voltage, PDMS thickness and the substrate material on mixing efficiency. The injection of two liquids was stopped during mixing, which was different from the mixing characterisation of the two parallel-flowing streams in a microchannel in the literature (Ozcelik et al. 2014). The characterisation method of this study is more suitable for the application of mixing in bead-based immunoassay, where beads and samples were mixed without injection flow. Figure 5A shows the micrographs of the dye and PBST mixed at 5 $\mathrm{kHz}$ and $28 \mathrm{~V}$ for 0, 2, 16 and 38 seconds (see Supplementary video 3). Before mixing, the dye and PBST approximately occupied each half of the microchamber (Figure 5A left). After the piezo was activated, the two liquids were quickly mixed. After 38 seconds of mixing, the fluorescence intensity of the mixture in the microchamber was uniform, which was an indication of through mixing.

As shown in Figure 5B, during mixing, the standard deviation of fluorescence intensity (SDFI) in the microchamber becomes smaller over time and constant when the mixing is complete. Although the start values $(\mathrm{t}=0)$ of SDFI are greatly different among various mixing conditions, all curves fatten out at a certain value when the mixture is even.

To further investigate the mixing progress, the SDFI (y) curve is fitted to a first-order decay model as below:

$$
y=a e^{-b t}+c
$$


where $t$ is the mixing time; $a$ and $c$ are constants referred to as amplitude and offset, respectively; and $b$ is a constant revealing the mixing ability. The amplitude $(a)$ is related to i) the dye concentration, ii) ratio of dye and PBST in the microchamber, and iii) the exposure time. These factors may vary significantly from experiment to experiment, so amplitudes of different curves (Figure 5B) are hardly equal to each other. The offset $(c)$ is mostly related to the uneven illumination of the microchamber. Even when the mixture is homogeneous, the SDFI is not equal to zero if the illumination across the microchamber is not uniform. $b$ is a constant reflects the mixing efficiency. It is dependent on PDMS thickness, driving frequency and voltage of the piezo, bubble size, and other factors that may affect the mixing. For example, when the dye and PBST were mixed in the device with 1-mm thick PDMS top layer on a glass bottom at $5 \mathrm{kHz}$ and $28 \mathrm{~V}$, the constants of the decay model were $a=482.5 \pm 16.7$, $b=0.1401 \pm 0.006$ and $c=64.27 \pm 1.43$. The $R^{2}$ of this model is 0.991 (Figure 5C). 

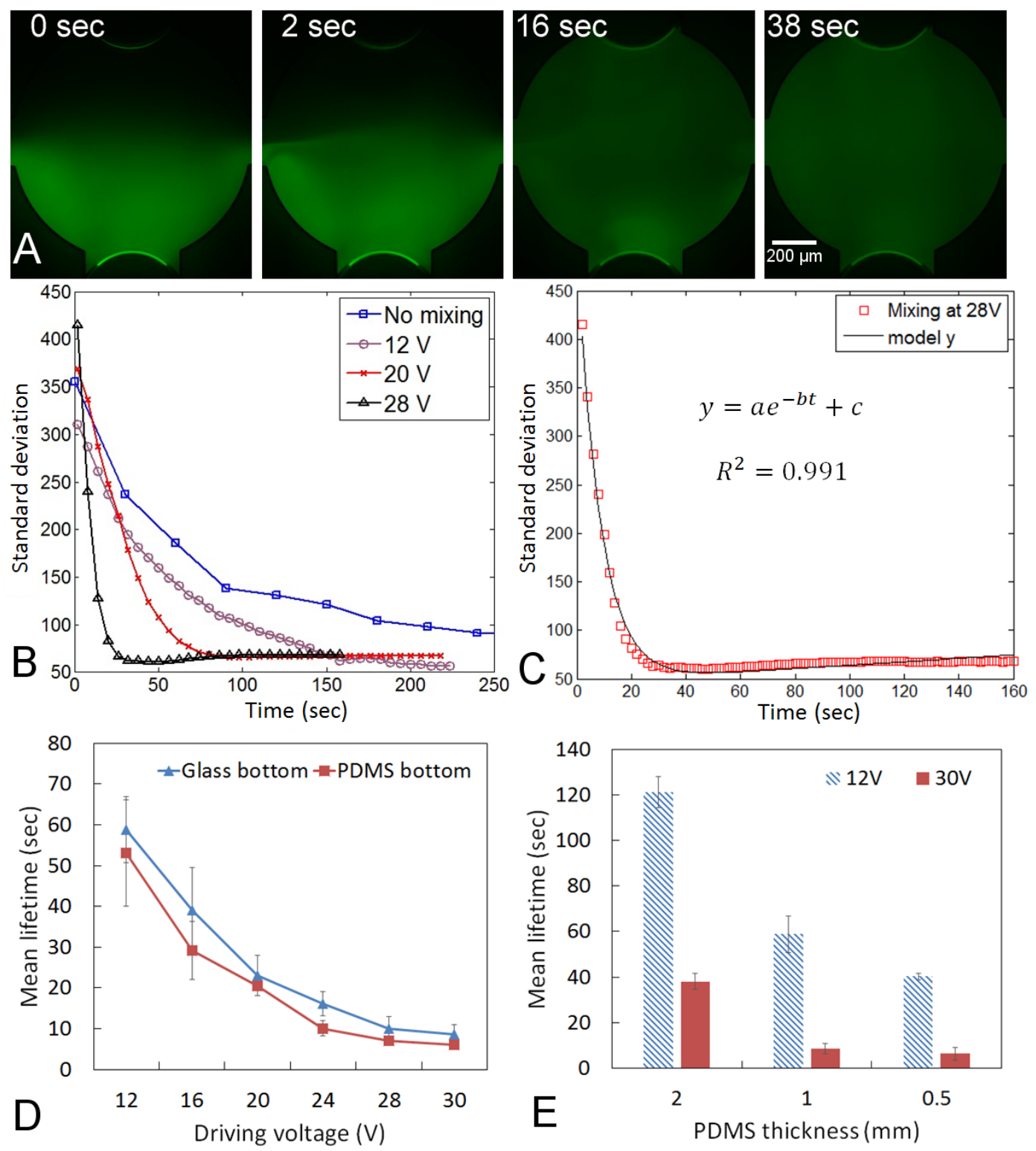

Figure 5 Mixing characterisation. A) Micrographs of dye and PBST mixed at the frequency of $5 \mathrm{kHz}$ and the driving voltage of $28 \mathrm{~V}$. B) Standard deviation of fluorescence intensity (SDFI) in the microchamber when dye and PBST were either not mixed (free diffusion) or mixed at 12, 20 and $28 \mathrm{~V}$ in the device with $1 \mathrm{~mm}$ thick PDMS top layer on glass bottom. C) SDFI was fitted to a first-order decay model. D) Effect of driving voltage on mean lifetime. E) Effect of PDMS layer thickness on mean lifetime.

For a standard first-order decay, the mean lifetime is given by:

$$
T=\frac{1}{h}
$$

where $b$ is the mixing constant given in Equation (8). Since the SDFI during mixing can be perfectly described by the first-order decay model (Equation (8)), the mean lifetime of SDFI is proposed to characterise the mixing efficiency. The smaller the mean lifetime is, the higher the mixing efficiency is. 
The mean lifetime when the dye and PBST were mixed at various driving voltages $(12 \mathrm{~V}-30 \mathrm{~V})$ in devices with either glass or PDMS bottom is shown in Figure 5D. It is inversely proportional to the driving voltage for both substrate materials. When the voltage is increased from $12 \mathrm{~V}$ to $30 \mathrm{~V}$, the mean lifetime decreases from 58.8 seconds to 8.7 seconds and from 53 seconds to 6.1 seconds for devices with glass and PDMS bottom, respectively. The mean lifetime of mixing in devices with PDMS bottom is slightly smaller than that in devices with glass bottom, regardless of the driving voltage. This difference may be related to the stiffness of the two substrates. In another word, the acoustic pressure in the bubble with PDMS bottom is probably bigger than that with a glass substrate. When the thickness of PDMS top layer (on glass bottom) varies from $2 \mathrm{~mm}$ to $1 \mathrm{~mm}$, the mean lifetime at $12 \mathrm{~V}$ and $30 \mathrm{~V}$ is reduced by $1 / 2$ and 1/4, respectively (Figure 5E). As the PDMS layer is decreased to $0.5 \mathrm{~mm}$ thick, the mean lifetime is further reduced to approximately two thirds of that with $1 \mathrm{~mm}$ thick PDMS layer.

Therefore this study reveals that the mixing efficiency is i) proportional to the driving voltage, ii) inversely proportional to the PDMS top layer thickness, and iii) higher for the devices with PDMS bottom in comparison to those with glass bottom.

The mean lifetime proposed in this study excludes the influence from both amplitude and the offset. It is able to better reflect the mixing efficiency compared to the mixing index, which has been widely applied to characterise the mixing efficiency (Huh et al. 2008; Lu et al. 2002; Ozcelik et al. 2014). The mixing index was defined by:

$$
\text { Mixing index }=\sqrt{N^{-1} \sum\left(\frac{c_{k}-\bar{c}}{\bar{c}}\right)^{2}}
$$

where $c_{k}$ is the colour index of pixel $\mathrm{k}$, and $\bar{c}$ is the average of the colour index over $N$ pixel on a line or in a region. The mixing index is actually the coefficient of variation of $N$ pixels. However, this method fails to eliminate the standard deviation of the colour of all pixels 
caused by uneven illumination. Additionally, it can hardly compare the efficiency of mixing for different experiments when the imaging conditions (such as light intensity and the exposure time) and the ratio of dye/PBST vary.

\subsection{Disaggregation of beads}

Following the manual injection of the beads solution, approximately 800 individual beads (counted using ImageJ) were left in the microchamber. A magnetic field of $0.11 \mathrm{~T}$, generated from permanent magnets positioned in the proximity of the mixing chamber, activated the movement of the magnetic beads. Because of the magnetic field in the mixing chamber and the dipole-dipole interactions, the beads formed clusters on the edge of the mixing chamber (see Figure $6 \mathrm{~A}$ ). After the activation of acoustic flow at $30 \mathrm{~V}$ and $5 \mathrm{kHz}$, the beads clumps were broken into individual beads in less than 3 seconds (see supplementary video 4). Figure $6 \mathrm{~B}$ and $\mathrm{C}$ show the dispersed single beads in the microchamber 30 and 55 seconds after the acoustic flow. Therefore, the acoustic microstreaming can not only disaggregate the bead clumps but homogeneously disperse the single beads in the microchamber, which is favourable for bead-based immunoassay in microfluidics. 

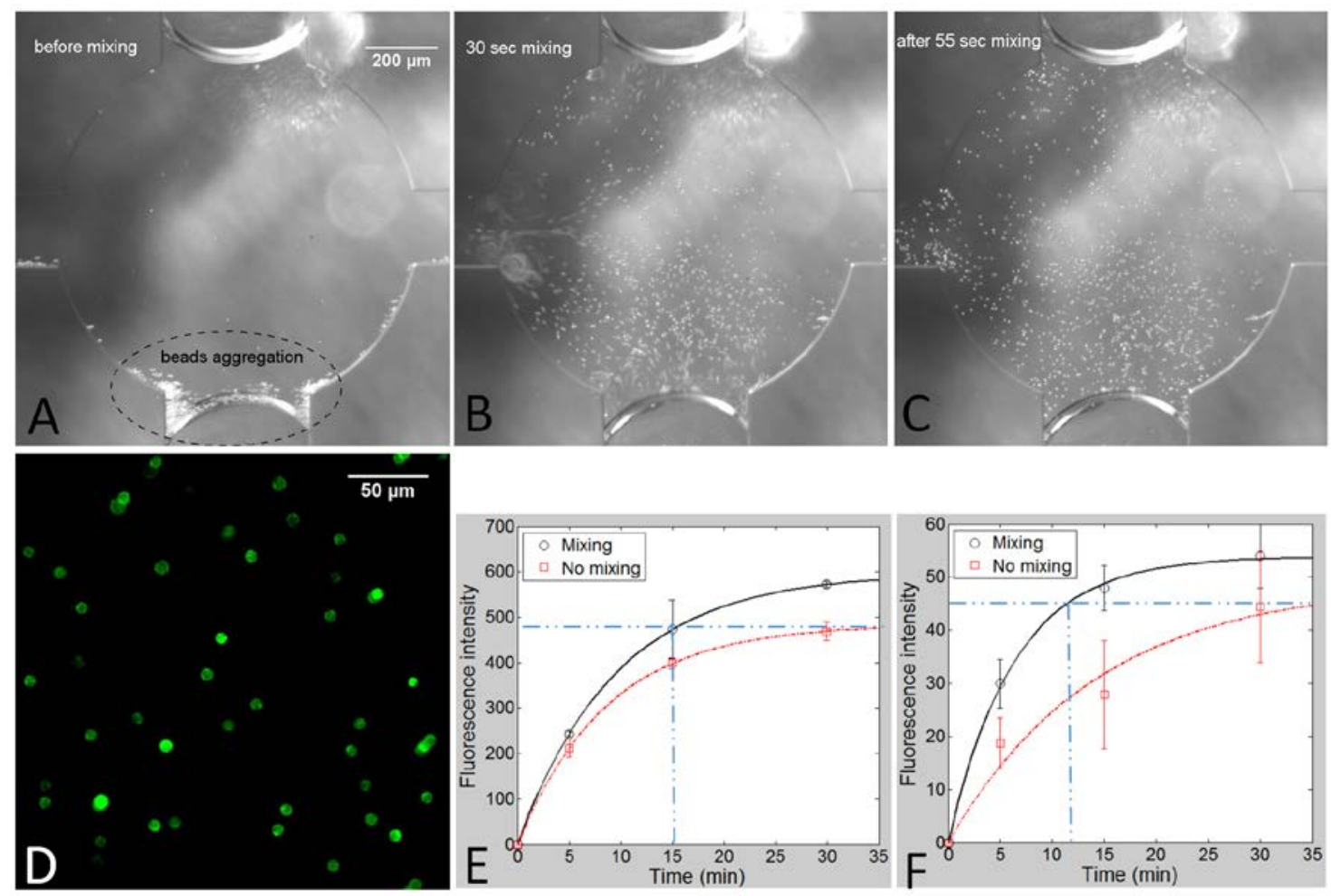

Figure 6 Disaggregation of beads and the on-chip immunoassay enhanced by acoustic micromixing. Micrographs of (A) bead clumps before mixing, (B) individual beads after the acoustic flow for 30 seconds, and (C) single beads 55 seconds after the acoustic flow. D) Micrograph of the beads at the end of the on-chip assay. Fluorescence intensities of beads at different incubation/mixing time for PSA concentration of $100 \mathrm{ng} / \mathrm{mL}$ (E) and $5 \mathrm{ng} / \mathrm{mL}$ (F). The black and red dotted lines represent the exponential growth models for the data with and without mixing, respectively.

Previous reports have shown aggregation of beads along the direction of external magnetic field (Cao et al. 2014; Marmottant and Hilgenfeldt 2004). Preventing beads from forming clusters or chains is important for maintaining the sensitivity and reproducibility of immunoassay in microfluidics. The active surface area of beads is maximal when they are physically away from each other. However, it is greatly reduced when beads form clusters. Consequently, the bead-surface-area-to-sample-volume ratio varies between different experimental runs, which may greatly reduce the reproducibility of analysis. Gao et al, reported the disaggregation of clusters with up to 60 beads using eight external electromagnets (Silvestre et al. 2013). 75\% of the clustered beads were reported to be dispersed in 50 seconds using this technique (Silvestre et al. 2013). In contrast, the acoustic mixing in this study efficiently dispersed a cluster with around 800 beads into individual beads in less than 3 seconds. After 55 seconds, the beads were homogeneously scattered over 
the whole microchamber by the acoustic microstreaming. The maximal surface area of the beads is able to increase the ability of capturing analyte, especially when the concentration of the analyte is low.

\subsection{On-chip immunoassay}

The PSA immunoassay was employed to demonstrate the capability of acoustic micromixing in reducing assay time. A concentration of $5 \mathrm{ng} / \mathrm{ml}$ was used for the PSA solution, which is clinically relevant since the normal range cut off value of PSA in human serum is $4 \mathrm{ng} / \mathrm{mL}$ (Brawer et al. 1992). For comparison, a higher concentration of $100 \mathrm{ng} / \mathrm{ml}$ was also studied. The PSA solutions were incubated with beads in the microfluidic device either at a static condition or with mixing for 5,15 or 30 minutes, respectively. The subsequent mixing or static incubation of the beads with R-PE label was fixed at 5 minutes. The total assay time was 15-40 minutes including 1-minute sample/R-PE label injection, 4-minute washing, 5 to 30-minute incubation of sample and 5-minute incubation of fluorescence label.

Supplementary video 5 shows the mixing of $5 \mu \mathrm{m}$ beads and PSA solution in the microchamber at $5 \mathrm{kHz}$ and $12 \mathrm{~V}$. The acoustic mixing is able to induce not only the flow of the liquid but also the movement of the beads. As a result of such motions, the beads gain more opportunity to actively capture PSA molecules in the solution. Figure 6D is the fluorescent micrograph of the beads dispersed homogeneously using the acoustic microstreaming. The fluorescence intensity of beads at the end of the assay is shown in Figures $6 \mathrm{E}$ and $\mathrm{F}$. At least 300 beads $(\mathrm{N}>300)$ at each time point are analysed to acquire the statistical significance. From Figures $6 \mathrm{E}$ and $6 \mathrm{~F}$, for both PSA concentrations, the micromixing can effectively enhance the assay, as reflected by the increase of the fluorescence intensity with mixing in comparison to that obtained without mixing. However, the increase is less pronounced for the high concentration PSA solutions. This is due to the 
more abundant PSA molecules in the solution and thus the active mixing is relatively less obvious.

The change of the fluorescence intensity with reaction time can be described by an exponential growth model: $y=a\left(1-e^{-b t}\right)$, where $a$ and $b$ are constants, and $t$ is the reaction time. For the PSA solution with a concentration of $100 \mathrm{ng} / \mathrm{ml}, a$ and $b$ have the values of 598 and $0.105\left(R^{2}=1\right)$ for the assay with mixing and 484.9 and $0.115\left(R^{2}=1\right)$ for the assay without mixing, respectively. For the PSA solution with a concentration of $5 \mathrm{ng} / \mathrm{ml}, a$ and $b$ have the values of 53.87 and $0.158\left(R^{2}=1\right)$ for the assay with mixing and 48.94 and $0.07\left(R^{2}=0.97\right)$ for the assay without mixing, respectively. It can be seen from Figures $6 \mathrm{E}$ and $\mathrm{F}$, when the acoustic micromixing is applied, the assays with $100 \mathrm{ng} / \mathrm{ml}$ and $5 \mathrm{ng} / \mathrm{ml}$ PSA solutions only take approximately 15 minutes and 12 minutes, respectively, to reach the same fluorescence intensity levels as those obtained with only static incubation for 35 minutes. The assay time was reduced by approximately 2.3 and 3.1 times for the high and low PSA concentrations, respectively. A similar finding has also been reported in previous publications (Gao et al. 2015; Petkovic-Duran et al. 2009; Petkovic et al. 2017). Such a mixing technique has been demonstrated with the capability of significantly speeding up immunoassays and shows the potential in lab on a chip devices for rapid detection of analyte.

\section{Conclusions}

This paper studies a technique to generate bubble-induced acoustic streaming in a microfluidic device for applications with microbeads based immunoassay. The bubbles are generated using a trapping method and, when an acoustic field is applied, induce microstreaming of fluids in the assay chamber. Such a microstreaming can not only effectively disperse beads to prevent aggregation but also generate mixing to speed up assay process. It is found that the Weber number should be in the range of $3.5 \times 10^{-7}$ to 0.087 to 
generate bubbles with reproducible size. Wider traps are suggested since $200-\mu \mathrm{m}$ and $400-\mu \mathrm{m}$ wide bubbles can be reliably controlled by hydraulic pressure in the range of 294 to $735 \mathrm{~Pa}$. The study also reveals that the mixing efficiency is proportional to the driving voltage and inversely proportional to the PDMS top layer thickness. The PDMS substrate slightly increases the mixing efficiency compared to the glass substrate. It has been demonstrated that the acoustic microstreaming can speed up the immunoassay by at least 2-3 times which shows great potential in application in lab on a chip devices for fast analysis of analytes.

\section{Acknowledgement}

This work was performed in part at the Microfluidic lab, Commonwealth Scientific and Industrial Organisation (CSIRO) and the Melbourne Centre for Nanofabrication (MCN) in the Victorian Node of the Australian National Fabrication Facility (ANFF). The support of the Center for Microfluidics and Nanofluidics of Harbin Institute of Technology (Shenzhen) is also greatly acknowledged.

\section{Competing interests}

The authors declare no competing financial interests. 


\section{References}

Ahmed D, Mao X, Juluri B, Huang T (2009a) A fast microfluidic mixer based on acoustically driven sidewalltrapped microbubbles Microfluidics and Nanofluidics 7:727-731 doi:10.1007/s10404-009-0444-3

Ahmed D, Mao X, Shi J, Juluri BK, Huang TJ (2009b) A millisecond micromixer via single-bubble-based acoustic streaming Lab on a Chip 9:2738-2741 doi:10.1039/B903687C

Bau HH, Zhong JH, Yi MQ (2001) A minute magneto hydro dynamic (MHD) mixer Sensors and Actuators BChemical 79:207-215 doi:10.1016/s0925-4005(01)00851-6

Brawer MK, Chetner MP, Beatie J, Buchner DM, Vessella RL, Lange PH (1992) SCREENING FOR PROSTATIC-CARCINOMA WITH PROSTATE SPECIFIC ANTIGEN Journal of Urology 147:841845

Cao QL, Han XT, Li L (2014) Configurations and control of magnetic fields for manipulating magnetic particles in microfluidic applications: magnet systems and manipulation mechanisms Lab on a Chip 14:27622777 doi:10.1039/c4lc00367e

Capretto L, Cheng W, Hill M, Zhang X (2011) Micromixing Within Microfluidic Devices. In: Lin BC (ed) Microfluidics: Technologies and Applications, vol 304. Topics in Current Chemistry. pp 27-68. doi:10.1007/128_2011_150

Chen HY, Li JJ, Zhang H, Li MS, Rosengarten G, Nordon RE (2011) Microwell perfusion array for highthroughput, long-term imaging of clonal growth Biomicrofluidics 5 doi:10.1063/1.3669371

Chen HY, Rosengarten G, Li MS, Nordon RE (2012) Design of microdevices for long-term live cell imaging Journal of Micromechanics and Microengineering 22 doi:10.1088/0960-1317/22/6/065033

Chindam C, Nama N, Ian Lapsley M, Costanzo F, Jun Huang T (2013) Theory and experiment on resonant frequencies of liquid-air interfaces trapped in microfluidic devices Journal of Applied Physics 114:194503 doi:10.1063/1.4827425

Delamarche E, Bernard A, Schmid H, Bietsch A, Michel B, Biebuyck H (1998) Microfluidic Networks for Chemical Patterning of Substrates: Design and Application to Bioassays Journal of the American Chemical Society 120:500-508 doi:10.1021/ja973071f

Gao Y, Tran P, Petkovic-Duran K, Swallow T, Zhu Y (2015) Acoustic micromixing increases antibody-antigen binding in immunoassays Biomedical microdevices 17:79 doi:10.1007/s10544-015-9987-0

Guo SS, Lau ST, Lam KH, Deng YL, Zhao XZ, Chen Y, Chan HLW (2008) Integration of ultrasonic transducers in fast prototyping microfluidic devices Journal of Applied Physics 103 doi:10.1063/1.2912825

Hai NH, Luong NH, Chau N, Tai NQ (2009) Preparation of magnetic nanoparticles embedded in polystyrene microspheres Journal of Physics: Conference Series 187:012009

Huang H-Y et al. (2015) Digital Microfluidic Dynamic Culture of Mammalian Embryos on an Electrowetting on Dielectric (EWOD) Chip PLoS ONE 10:e0124196 doi:10.1371/journal.pone.0124196

Huh YS, Park TJ, Lee EZ, Hong WH, Lee SY (2008) Development of a fully integrated microfluidic system for sensing infectious viral disease Electrophoresis 29:2960-2969 doi:10.1002/elps.200700823

Jo MC, Guldiken R (2013) Dual surface acoustic wave-based active mixing in a microfluidic channel Sensors and Actuators A: Physical 196:1-7 doi:http://dx.doi.org/10.1016/j.sna.2013.03.028

Kang JH, Kim YC, Park J-K (2008) Analysis of pressure-driven air bubble elimination in a microfluidic device Lab on a Chip 8:176-178 doi:10.1039/b712672g

Kevin W, Fan ZH (2015) Mixing in microfluidic devices and enhancement methods Journal of Micromechanics and Microengineering 25:094001

Kitenbergs G, Erglis K, Perzynski R, Cebers A (2015) Magnetic particle mixing with magnetic microconvection for microfluidics Journal of Magnetism and Magnetic Materials 380:227-230 doi:10.1016/j.jmmm.2014.10.033

Lee CY, Chang CL, Wang YN, Fu LM (2011) Microfluidic Mixing: A Review International Journal of Molecular Sciences 12:3263-3287 doi:10.3390/ijms12053263

Lin Y-H, Wang C-C, Lei KF (2014) Bubble-driven mixer integrated with a microfluidic bead-based ELISA for rapid bladder cancer biomarker detection Biomedical microdevices 16:199-207 doi:10.1007/s10544013-9822-4

Liu RH, Lenigk R, Druyor-Sanchez RL, Yang J, Grodzinski P (2003) Hybridization Enhancement Using Cavitation Microstreaming Analytical chemistry 75:1911-1917 doi:10.1021/ac026267t 
Liu RH, Yang J, Pindera MZ, Athavale M, Grodzinski P (2002) Bubble-induced acoustic micromixing Lab on a Chip 2:151-157 doi:10.1039/B201952C

Liu RH, Yang JN, Lenigk R, Bonanno J, Grodzinski P (2004) Self-contained, fully integrated biochip for sample preparation, polymerase chain reaction amplification, and DNA microarray detection Analytical chemistry 76:1824-1831 doi:10.1021/ac0353029

Lu LH, Ryu KS, Liu C (2002) A magnetic microstirrer and array for microfluidic mixing Journal of Microelectromechanical Systems 11:462-469 doi:10.1109/JMEMS.2002.802899

Marmottant P, Hilgenfeldt S (2004) A bubble-driven microfluidic transport element for bioengineering Proceedings of the National Academy of Sciences of the United States of America 101:9523-9527 doi:10.1073/pnas.02307007101

Oddy MH, Santiago JG, Mikkelsen JC (2001) Electrokinetic instability micromixing Analytical chemistry 73:5822-5832 doi:10.1021/ac0155411

Ozcelik A, Ahmed D, Xie Y, Nama N, Qu Z, Nawaz AA, Huang TJ (2014) An Acoustofluidic Micromixer via Bubble Inception and Cavitation from Microchannel Sidewalls Analytical chemistry 86:5083-5088 doi:10.1021/ac5007798

Pamme N (2012) On-chip bioanalysis with magnetic particles Curr Opin Chem Biol 16:436-443 doi:10.1016/j.cbpa.2012.05.181

Patel MV, Nanayakkara IA, Simon MG, Lee AP (2014) Cavity-induced microstreaming for simultaneous onchip pumping and size-based separation of cells and particles Lab on a Chip 14:3860-3872 doi:10.1039/c4lc00447g

Patel MV, Tovar AR, Lee AP (2012) Lateral cavity acoustic transducer as an on-chip cell/particle microfluidic switch Lab on a Chip 12:139-145 doi:10.1039/C1LC20626E

Petkovic-Duran K, Manasseh R, Zhu Y, Ooi A (2009) Chaotic micromixing in open wells using audiofrequency acoustic microstreaming BioTechniques 47:827-831 doi:10.2144/000113242

Petkovic K, Metcalfe G, Chen H, Gao Y, Best M, Lester D, Zhu Y (2017) Rapid detection of Hendra virus antibodies: an integrated device with nanoparticle assay and chaotic micromixing Lab on a Chip 17:169-177 doi:10.1039/C6LC01263A

Riahi M, Alizadeh E (2012) Fabrication of a 3D active mixer based on deformable Fe-doped PDMS cones with magnetic actuation Journal of Micromechanics and Microengineering 22 doi:Artn 115001

10.1088/0960-1317/22/11/115001

Rida A, Gijs MAM (2004) Manipulation of Self-Assembled Structures of Magnetic Beads for Microfluidic Mixing and Assaying Analytical chemistry 76:6239-6246 doi:10.1021/ac049415j

Schneider CA, Rasband WS, Eliceiri KW (2012) NIH Image to ImageJ: 25 years of image analysis Nat Meth 9:671-675

Shilton RJ, Yeo LY, Friend JR (2011) Quantification of surface acoustic wave induced chaotic mixing-flows in microfluidic wells Sensors and Actuators B: Chemical 160:1565-1572 doi:http://dx.doi.org/10.1016/j.snb.2011.09.007

Silvestre ME, Franzreb M, Weidler PG, Shekhah O, Wöll C (2013) Magnetic Cores with Porous Coatings: Growth of Metal-Organic Frameworks on Particles Using Liquid Phase Epitaxy Advanced Functional Materials 23:1210-1213 doi:10.1002/adfm.201202078

Stroock AD, Dertinger SKW, Ajdari A, Mezic I, Stone HA, Whitesides GM (2002) Chaotic mixer for microchannels Science 295:647-651 doi:10.1126/science.1066238

van Reenen A, de Jong AM, den Toonder JMJ, Prins MWJ (2014) Integrated lab-on-chip biosensing systems based on magnetic particle actuation - a comprehensive review Lab on a Chip 14:1966-1986 doi:10.1039/c3lc51454d

Veetil JV, Jin S, Ye K (2010) A glucose sensor protein for continuous glucose monitoring Biosensors \& Bioelectronics 26:1650-1655 doi:10.1016/j.bios.2010.08.052

Volk A, Rossi M, Kaehler CJ, Hilgenfeldt S, Marin A (2015) Growth control of sessile microbubbles in PDMS devices Lab on a Chip 15:4607-4613 doi:10.1039/c5lc00982k

Wang W-c, Peng C, Shi K, Pan Y-x, Zhang H-s, Ji X-l (2014) Double emulsion droplets as microreactors for synthesis of magnetic macroporous polymer beads Chin J Polym Sci 32:1639-1645 doi:10.1007/s10118-014-1543-5

Wang Y, Zhe J, Chung BTF, Dutta P (2008) A rapid magnetic particle driven micromixer Microfluidics and Nanofluidics 4:375-389 doi:10.1007/s10404-007-0188-x

Yaralioglu GG, Wygant IO, Marentis TC, Khuri-Yakub BT (2004) Ultrasonic mixing in microfluidic channels using integrated transducers Analytical chemistry 76:3694-3698 doi:10.1021/ac035220k

Zolgharni M, Azimi SM, Bahmanyar MR, Balachandran W (2007) A numerical design study of chaotic mixing of magnetic particles in a microfluidic bio-separator Microfluidics and Nanofluidics 3:677-687 doi:10.1007/s10404-007-0160-9 